\title{
Topological protection of biphoton states
}

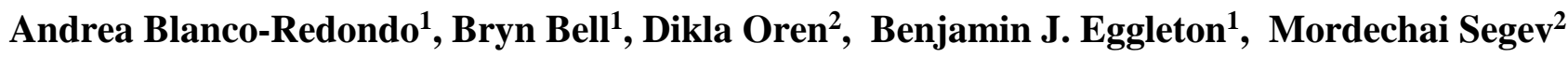

1. Institute of Photonics and Optical Science (IPOS), The Sydney Nano Institute, School of Physics, The University of Sydney, Sydney, New South Wales 2006, Australia.

2. Physics Department and Solid State Institute, Technion-Israel Institute of Technology, Haifa 32000, Israel

Robust generation and propagation of multiphoton quantum states are crucial for applications in quantum information, computing and communications. While photons are intrinsically well isolated from the thermal environment, scaling to large quantum optical devices is still limited by scattering loss and other errors arising from random fabrication imperfections. The recent discoveries on topological phases have introduced avenues to construct quantum systems that are protected against scattering and imperfections. We experimentally demonstrate topological protection of biphoton states, the building block for quantum information systems. We provide clear evidence of robustness of the spatial features and the propagation constant of biphoton states generated within a nanophotonics lattice with non-trivial topology and propose a concrete path to build robust entangled states for quantum gates.

The past decade has witnessed discoveries on topological states of matter, impacting fields beyond condensed matter, such as electromagnetism (1) and photonics (2-4), acoustics (5), cold atoms (6,7), and mechanics (8). The reasons for this extensive research interest are twofold: (i) the underlying concepts are fundamental and universal to many wave systems in nature, and (ii) the topological features of these systems offer the possibility of topological protection of transport for classical and quantum waves.

In photonics specifically, even though multiphoton quantum states have played a major role in testing quantum mechanics, quantum computing and communications, the scalability to large quantum optical devices is limited by loss and fabrication imperfections (9). With the major progress in topological photonics, experiments looking at transport of quantum edge states using single photons 
have been recently carried out (10-12), of clear interest for quantum simulation and sensing. These single-photon experiments studied the physics of topologically protected bounds states (10) and topological transitions (11) in photonic quantum walks, as well as demonstrating an interface between a quantum emitter and a photonic topological edge state (12). However, quantum information systems rely on multiphoton states, thus highlighting the importance of demonstrating topological robustness of multiphoton states. It has been theoretically suggested that topological features can provide robustness to the transport of biphoton states (13-15), and, recent experiments have shown that topology can lead to robustness in the spectral correlations between photon pairs (16).

Our experimental system is a one-dimensional (1D) array of silicon nanowires (Fig. 1A) comprising alternating short and long gaps that modulate the coupling strength between adjacent nanowires creating a Su, Schrieffer, Heeger (SSH) lattice $(17,18)$. A long-long defect in the middle of the lattice acts as a topological interface between two mirror-reflection versions of the SSH lattice, yielding a topological edge state at the edge to both (19). Given the topological nature of the SSH lattice, two key features of this mode are protected against any disorder that preserves the chiral symmetry of the system: the propagation constant (analogous to the energy condensed matter systems) and the zeroamplitude of the wave function in every other site.

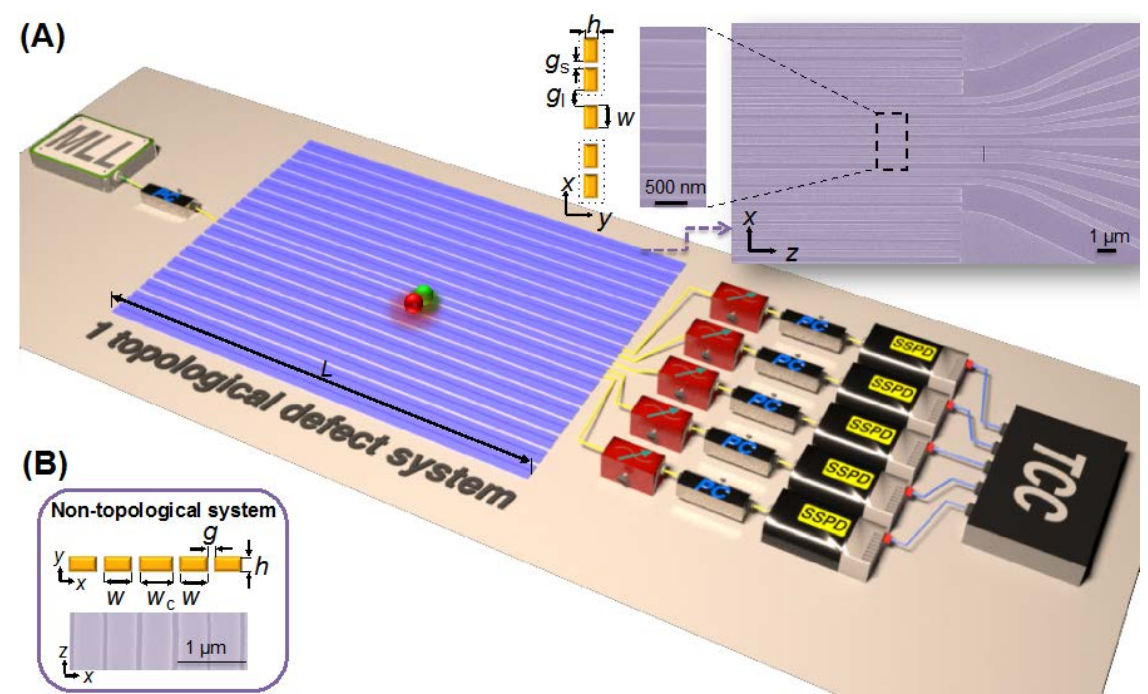

Fig. 1. Our nanophotonics topological system and experimental setup. (A) Mode-locked laser (MLL), dimer chain of silicon nanowires with one topological (long-long) defect, polarization controllers (PC), tunable filters, superconducting single-photon detectors (SSPD), and time correlation circuit (TCC). Inset: SEM of the topological defect. (B) SEM of the center waveguides of the structure providing non-topological (index-guiding) localization. (SEM images credit: Guanghui Ren, RMIT University). 
Intense picosecond pump pulses from a mode-locked laser (MLL) at $1550 \mathrm{~nm}$ are focused into the waveguide at the center of the lattice (Fig. 1A). As the pulses propagate in the lattice, they efficiently generate correlated signal and idler photons over a broad bandwidth via spontaneous four-wave mixing (SFWM) (20). After the chip, we filter signal and idler photons at $1545 \mathrm{~nm}$ and $1555 \mathrm{~nm}$ respectively. We detect individual photons using superconducting single-photon detectors (SSPDs) and identify matching arrival times using a time correlation circuit (TCC). In this way, we measure the spatial profile of the wavefunction and the biphoton correlations as a function of the lattice position.

Generally, photon pairs generated within a lattice have entanglement between the spatial modes of the lattice (in addition to frequency entanglement) (21). In our lattice, the non-trivial topology provides additional selection rules to the generation of the biphoton states (22), in addition to the phasematching condition typical of trivial quantum walks of correlated photons based on SFWM. Since the pump beam excites the topological defect mode, it stays confined to the defect. As such, the biphotons strongly overlap with the wavefunction of topological defect mode. This yields topological protection of several features of the biphototon wavefunction, and at the same time makes the wavefunction close to - but not exactly - separable.

This underlies an important difference between the current experiments with biphoton states and previous topological photonic studies that use classical light. While the eigenmodes of the structure are the same as for classical states, the wavefunctions of multiphoton states exist in a higher-dimensional Hilbert space - in particular the current experiments with two non-identical photons in $m$ waveguides exist in a $\mathrm{m}^{2}$ dimensional Hilbert space. The presence of the topological defect state localizes the biphoton to a near-separable state, but a $\mathrm{m}^{2}$ dimensional Hilbert space is still required to describe the state because signal and idler cannot be described independently, even if the photons are non-identical (23). In this context, previous topological experiments using single-photon states (10-12), which live in an $m^{1}$ dimensional Hilbert space, are similar to those using their corresponding classical states. 
Figure 1A shows a scanning electron micrograph (SEM) of the fabricated topological lattice of silicon nanowires on a silica substrate (Fig. 1A), where $h=220 \mathrm{~nm}, w=450 \mathrm{~nm}, g_{\mathrm{s}}=173 \mathrm{~nm}, g_{1}=307$ nm (20). The lattice consists of 203 nanowires with length $L=381 \mu \mathrm{m}$. The center nanowires fan out at the output of the array to allow the collection of photons via grating coupling to a fiber array. For comparison, we also fabricate a non-topological lattice of equidistant nanowires with a wider center waveguide (Fig. 1B), giving rise to a localized topologically-trivial defect mode. Choosing $g=100 \mathrm{~nm}$ and $w_{\mathrm{s}}=465 \mathrm{~nm}$, we achieve the same confinement at the 5 center waveguides for the topologically and trivially localized defect modes.

To experimentally demonstrate the robustness of the topological biphoton state against disorder, we fabricate and measure structures without disorder and with deliberately introduced random disorder in the position of the waveguides and thus in the coupling constants. In our nanophotonics platform, randomizing the positions of the center of each nanowire within a range of $\pm 14 \mathrm{~nm}$ (respectively \pm 43 $\mathrm{nm}$ ) results in disorder of $20 \%$ (respectively $60 \%$ ) of the original coupling constant.

We measure biphoton correlations (Fig. 2) over 120 seconds at the output of the five center waveguides of the topological (A)-(C) and the non-topological lattice (D)-(F), for increasing levels of disorder. Here we are measuring individual structures, exemplifying a particular instance of random disorder. The measurements agree well with the quantum states resulting from the propagation simulations at each level of disorder (Supplementary Videos). The vertical scale is unique for each measured structure and shows the true signal and idler coincidence counts between waveguides, having subtracted the background level of accidental coincidences. The coincidence to accidental ratio (CAR) is well above 10 for all significant points in the correlation matrix.

For the biphoton quantum state in the topological lattice, the strong center peak in Figs. 2A-C shows that, with high probability, both correlated photons are in waveguide 0 even for very high levels of disorder. This is evidence of the strong localization of the quantum state at the topological defect. However, the most crucial feature in this topological quantum state is that the counts remain zero in the odd-waveguides. The robustness of this quantum state stems from its strong overlap with the topological 
edge mode of the lattice, whose wavefunction is immune to disorder (Fig. 3A). The Schmidt number (K) of the measured topological biphoton states, which is essentially a witness of the mode entanglement, is not protected against disorder, as shown in Fig. 2. Nevertheless, $K$ remains close to 1 because the topology guarantees the presence of a single localized mode, even with disorder, and the biphotons are well-overlapped with this mode.

(A)

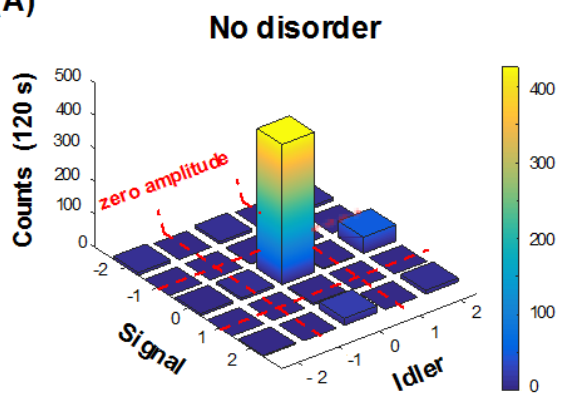

(D)

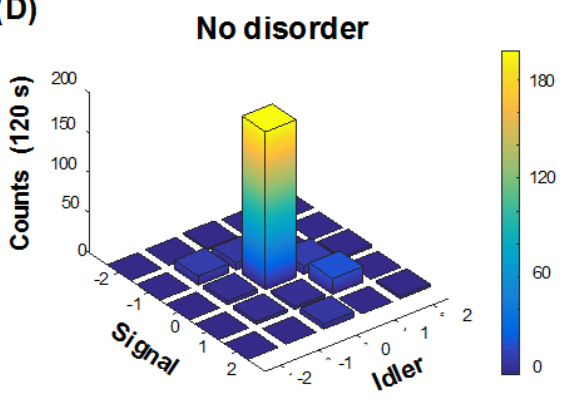

(B)

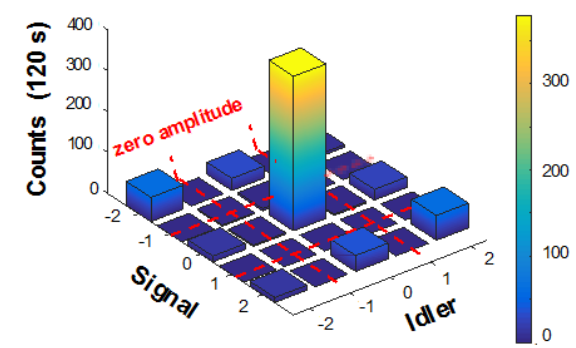

(E)

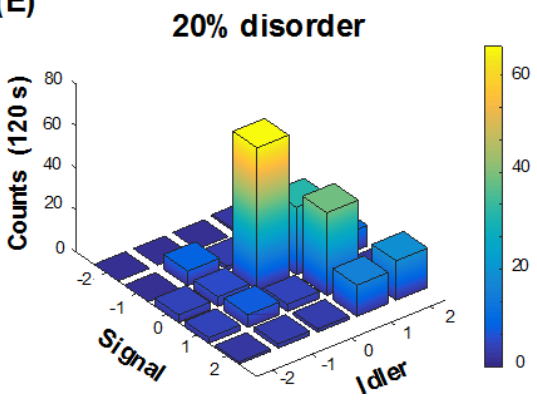

(C)

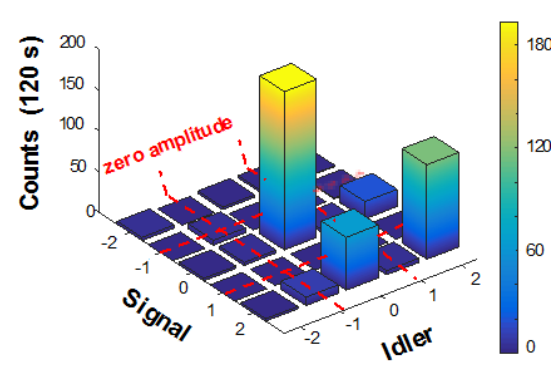

(F)

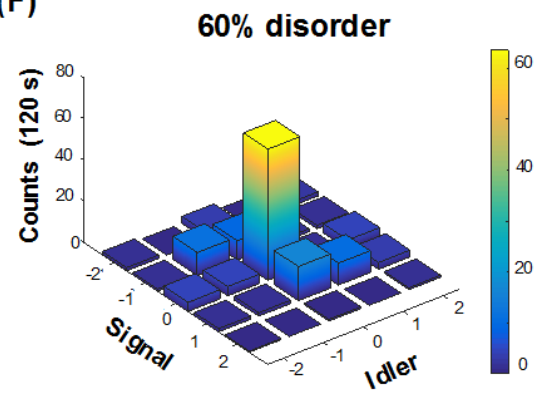

Fig. 2. Measured biphoton states with and without disorder. (A)-(C) Detected biphoton correlations of topological quantum state for random disorder in a range of $0 \%, 20 \%$ and $60 \%$ in the coupling constants: the probability amplitude remains zero in the odd-waveguides. (D)-(F) Detected biphoton correlations of non-topological quantum state in the same conditions of disorder. The disorder causes all coincidence amplitudes to vary, and no feature is preserved.

These measurements beg the question: are the quantum correlations playing a crucial role or is the robustness observed here just a logical consequence of the topological protection of classical light (20) or of single photons $(12,13)$ in the SSH model? We calculate the value of following quantity

$$
\Gamma_{i j}^{\Delta, s i}=\left\langle n_{i}^{s} n_{j}^{i}\right\rangle-\left\langle n_{i}^{s}\right\rangle\left\langle n_{j}^{i}\right\rangle
$$

where the term $\left\langle n_{i}^{s} n_{j}^{i}\right\rangle$ denotes the signal-idler correlations and the term $\left\langle n_{i}^{s}\right\rangle\left\langle n_{j}^{i}\right\rangle$ denotes the product of the signal and idler intensities in waveguides $i$ and $j$ respectively. Our calculation shows that $\Gamma_{i j}^{\Delta, s i}$ is almost entirely determined by the biphoton correlation (20). Therefore, the measurements in Fig. 2, 
reveal an unexplored regime beyond the classical topological protection of electromagnetic states, with new quantities coming into play and new aspects of the wavefunction being protected.

The topological edge states in the SSH model are protected against any kind of disorder that preserves the chirality. In our nanophotonics SSH platform this includes disorder in the position of the waveguides, environmental changes resulting in a $z$-uniform refractive index change, and wavelength fluctuations. However, a small amount of disorder in the width of the individual waveguides, which does not respect the lattice chirality, is inevitable due to fabrication precision limits. This is the cause of the only minor outlier in our measurements, the detection of a small but non-vanishing number of coincidences between waveguides 2 and -1 in Fig. 2C. Due to fabrication imperfections, waveguide -1 is slightly wider than the rest in this particular device, leading to slight index localization.

For comparison, we repeat the same set of measurements for the non-topological lattice described above. The measured biphoton states in Fig. 2D-F show localization of the biphoton state around the center waveguide but reveal severe delocalization even at low levels of disorder. More importantly, there is no conserved experimental observable, even though the pump is launched at a defect mode. Because the defect in Fig. 2D-F is non-topological, the emerging biphoton state does not exhibit any feature that is conserved in the presence of disorder (Fig. 3B).
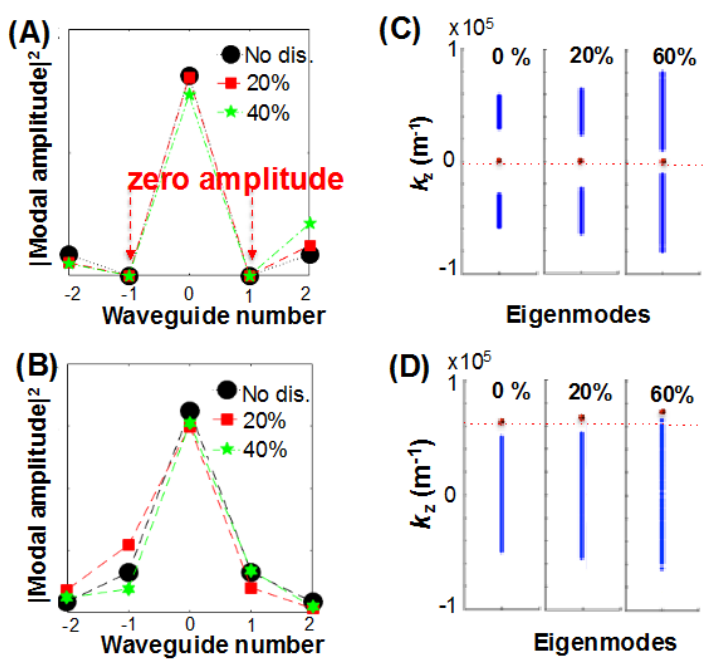

Fig. 3. Modal amplitudes and energies in the presence of lattice disorder. (A) Calculated amplitude squared of topological defect mode with $0 \%$ (black) $20 \%$ (red) and $40 \%$ (green) disorder, the modal amplitude remains zero in the odd waveguides for all levels of disorder. (B) Similar for trivial mode, however, here no feature is preserved. (C) and (D) Mode propagation constant $\left(k_{\mathrm{z}}\right)$ - equivalent to mode energy - for topological and trivial systems respectively. The red dot is $k_{\mathrm{z}}$ of the defect mode, which remains unchanged under disorder in the topological system but changes in the non-topological system. 
The other striking signature of topological protection in the SSH model is associated with the zeroes of the wave function: the energy of the topological edge mode (red dot) remains pinned at zero for all levels of disorder (Fig. 3C). In practice, this entails that the propagation constant $\left(k_{\mathrm{z}}\right)$ of the topological mode does not change with disorder, which implies that the phase imparted by the lattice on the quantum state is robust. In contrast, the propagation constant of the index-guided mode, red dot in Fig. 3D, varies appreciably even for low levels of disorder, leading to phase errors which accumulate to very large phase variations at the lattice output. This highlights the importance of the results presented here in the context of quantum information systems with entanglement between different modes.

Let us now show using simulations that the nanophotonic platform for topologically protected waveguiding of biphoton quantum states demonstrated here can be used as the key building block of an entangled system. We put together two of these building blocks in a 2-topological-defects system (Fig. 4A). This yields two topological defect modes that are uncoupled from each other provided that there is enough separation between defects $A$ and $B$. The entanglement between these two modes can be generated in the same lattice or, alternatively, one can use entangled states generated outside the chip as the input to the topological modes. Here, we simulate the generation of an entangled state between the two topological defect modes on-chip using two intense pumps at the center of each topological defect and SFWM (21). This topological two-mode system can serve as the building block of a variety of quantum gates in a quantum information system. Here we show how it could be used for a quantum gate operating on 2 qubits.

The interference between the quantum states emerging from the two topological defect modes of the lattice is robust against disorder. We simulate the biphoton generation and propagation with and without disorder, and the interference of the defect modes $A$ and $B$ at the output (Fig 4A), using a beam splitter. This interference generates four peaks, $\left|A>_{\mathrm{s}}\right| A>_{\mathrm{i}},\left|B>_{\mathrm{s}}\right| B>_{\mathrm{i}},\left|A>_{\mathrm{s}}\right| B>_{\mathrm{i}},\left|B>_{\mathrm{s}}\right| A>_{\mathrm{i}}$, representing the presence of signal and idler ( $s$ and $i$ ) in defect modes $A$ and $B$. At the output of the lattice, but before the interferometer, we have $\left|A>_{s}\right| A>_{i},\left|B>_{s}\right| B>_{i}$ corresponding to the entangled state in Fig. 4B. After the interferometer these two peaks disappear due to destructive interference and the peaks $\left|A>_{\mathrm{s}}\right| B>_{\mathrm{i}}\left|B>_{\mathrm{s}}\right| A>_{\mathrm{i}}$ 
appear, experiencing constructive interference (Fig. 4C). This is similar to reverse Hong-Ou-Mandel interference where entangled pairs of photons at the beam-splitter inputs are split and appear as one photon at each output.

Fig. 4. Protection of entanglement in a simulated 2-topological defect system. (A) Nanophotonics platform with two topological defects for generation and protection of entanglement. The output of modes A and B interfere in a beam splitter. (B-E) Simulated biphoton correlations before and after the beam splitter with no disorder and $20 \%$ disorder for the 2-topological defect system. (F-I) Similar for 2trivial defect system. The red circles in (I) indicate the (false) correlation peaks introduced by disorder.

In the presence of $20 \%$ disorder in the coupling constant, the propagation constant of the topological defect modes $A$ and $B, k_{\mathrm{z}, \mathrm{A}}$ and $k_{\mathrm{z}, \mathrm{B}}$, remain unchanged and thus the entanglement is preserved (Figs. 4D and E). This robustness should be compared to an equivalent system of two trivial (non- 
topological) defects, as the ones measured above. In the absence of disorder, our simulations show the two peaks before (Fig.4F) and after the interferometer (Fig. 4G) are similar to the topological case. However, in the presence of disorder $k_{\mathrm{z}, \mathrm{A}}$ and $k_{\mathrm{z}, \mathrm{B}}$ change, and the output phases are randomized, which leads to all four peaks present in the biphoton correlation after the beam splitter (Fig. 4I). When the topological protection is absent the entangled state takes on vastly different forms in the presence of disorder, such that on average (over multiple realizations of disorder) entanglement is lost. The concept of topology in this system, however, goes beyond making this platform more tolerant to fabrication defects. It offers a degree of freedom to generate new entangled quantum states in a multi-dimensional Hilbert-space in a CMOS-compatible platform.

The results presented here provide definitive experimental evidence of the protection that the underlying topology of the structure can provide to the spatial features of states living in highdimensional Hilbert spaces. We showed how the topological nanophotonic platform can be used to enable scalable entangled quantum information systems robust to fabrication disorder. Here, the multidimensionality of the wavefunction highlights new directions in topological physics, as new variables become relevant and can be affected by topology. As we have shown here, this is also a versatile platform to investigate novel phenomena combining topological edge modes with nonlinear optics, with the ability to generate and manipulate quantum correlations. Finally, the topological protection of the biphoton states demonstrated here for the one-dimensional SSH lattice serves as an experimental proof-of-concept for any other topological photonic quantum system, including twodimensional lattices such as (2-4) which should exhibit topologically-protected transport around the perimeter of the lattice, with protection against all kinds of disorder that does not break time-reversal symmetry. These findings lead to many new avenues in conjunction with boson sampling $(24,25)$, quantum simulations $(26,27)$, quantum computing (9), and a variety of exciting new possibilities related to the evolution of quantum states in photonic structures (28). 


\section{REFERENCES}

1. Z. Wang, Y. D. Chong, J. D. Joannopoulos, M. Soljačić, Observation of unidirectional backscattering-immune topological electromagnetic states. Nature 461, 772-775 (2009).

2. M. C. Rechtsman et al., Photonic Floquet topological insulators. Nature 496, 196-200 (2013).

3. M. Hafezi, S. Mittal, J. Fan, A. Migdall, J. M. Taylor, Imaging topological edge states in silicon photonics. Nat. Photonics 7, 1001-1005 (2013).

4. T. Ma, A. B. Khanikaev, S. H. Mousavi, G. Shvets, Guiding electromagnetic waves around sharp corners: topologically protected photonic transport in metawaveguides. Phys. Rev. Lett. 114, 127401 (2015)

5. Z. Yang et al., Topological acoustics. Phys. Rev. Lett. 114, 114301 (2015).

6. M. Aidelsburger et al., Measuring the Chern number of Hofstadter bands with ultracold bosonic atoms. Nat. Phys. 11, 162-166 (2014).

7. G. Jotzuet al., Experimental realization of the topological Haldane model with ultracold fermions. Nature 515, 237-240 (2014).

8. R. Süsstrunk, S. D. Huber, Observation of phononic helical edge states in a mechanical topological insulator. Science 349, 47-50 (2015).

9. A. Politi, J. Matthews, M. G. Thompson, J. L. O’Brien, Integrated quantum photonics. IEEE J. Sel. Top. Quantum Electron. 15, 1673-1684 (2009).

10. T. Kitagawa et al., Observation of topologically protected bound states in photonic quantum walks, Nat. Commun. 3, 882 (2012).

11. F. Cardano et al., Statistical moments of quantum-walk dynamics reveal topological quantum transitions, Nat. Commun. 7, 11439 (2016).

12. S. Barik et al., A topological quantum optics interface. Science 359 (6376), 666-668 (2018).

13. M. C. Rechtsman et al., Topological protection of photonic path entanglement. Optica 3, 925 (2016).

14. S. Mittal, V. V. Orre, M. Hafezi, Topologically robust transport of entangled photons in a 2D photonic system. Opt. Express 24, 15631 (2016).

15. M. A. Gorlach, A. N. Poddubny, Topological edge states of bound photon pairs. Phys. Rev. A 95, 53866 (2017).

16. S. Mittal, M. H. Hafezi, Topologically robust generation of correlated photon pairs. arXiv:1709.09984v1 [physics.optics]

17. W. P. Su, J. R. Schrieffer, A. J. Heeger, Solitons in polyacetylene. Phys. Rev. Lett. 42, 1698-1701 
(1979).

18. N. Malkova, I. Hromada, X. Wang, G. Bryant, Z. Chen, Observation of optical Shockley-like surface states in photonic superlattices. Opt. Lett. 34, 1633 (2009).

19. A. Blanco-Redondo et al., Topological optical waveguiding in silicon and the transition between topological and trivial defect states. Phys. Rev. Lett. 116, 163901 (2016). erratum 117, 129901 (2016).

20. Supplementary Material

21. A. Peruzzo et al., Quantum walks of correlated photons. Science 329, 1500-3 (2010).

22. D. Leykam, A. S. Solntsev, A. A. Sukhorukov, A. S. Desyatnikov, Lattice topology and spontaneous parametric down-conversion in quadratic nonlinear waveguide arrays. Phys. Rev. A 92, 33815 (2015).

23. A. S. Solntsev, A. A. Sukhorukov, D. N. Neshev, Y. S. Kivshar, Photon-pair generation in arrays of cubic nonlinear waveguides, Opt. Express 20, 27441-27446 (2012).

24. J. B. Spring et al., Boson Sampling on a Photonic Chip. Science 339, 798-801 (2013).

25. M. A. Broome et al., Photonic Boson Sampling in a Tunable Circuit. Science 339, 794-798 (2013).

26. M. S. Rudner, L.S. Levitov, Topological Transition in a Non-Hermitian Quantum Walk. Phys. Rev. Lett. 102, 65703 (2009).

27. J. M. Zeuner et al., Observation of a topological transition in the bulk of a non-Hermitian system. Phys. Rev. Lett. 115, 40402 (2015).

28. C. Reimer et al., Generation of multiphoton entangled quantum states by means of integrated frequency combs. Science 351, 1176-1180 (2016).

Acknowledgments: Funding: The authors gratefully acknowledge financial support from The Technion Society of Australia (NSW) and the NSW Department of Industry (The University of Sydney and the Technion collaborative photonics research project), the School of Physics of the University of Sydney (Professor Harry Messel Research Fellowship), the Australian Research Council (CE110001018, FL120100029). Author contributions: All authors contributed to all aspects of this work. Competing interests: The authors declare no competing financial interests. Data and materials availability: All data are available in the manuscript or the supplementary materials. 
Supplementary Materials for this manuscript include:

1. PDF file with:

Materials and Methods

Supplementary Text

Figs. S1 to S3

Captions for Movies S1 to S2

2. Movie S1

3. Movie S2 